EPJ Web of Conferences 37, 06013 (2012)

DOI: $10.1051 /$ epjconf/20123706013

(C) Owned by the authors, published by EDP Sciences, 2012

\title{
Meson Photoproduction Experiments with CLAS
}

\author{
Eugene Pasyuk $^{\mathrm{a}}$ and the CLAS Collaboration \\ Thomas Jefferson National Accelerator Facility, Newport News, VA, U.S.A.
}

\begin{abstract}
A large part of the experimental program in Hall B of the Jefferson Lab is dedicated to light baryon spectroscopy. Meson photoprodcution experiments are essential part of this program. CEBAF Large Acceptance Spectrometer (CLAS) and availability of circularly and linearly polarized tagged photon beams and frozen spin polarized targets provide unique conditions for this type of experiments. This combination of experimental tools gives a remarkable opportunity to measure double polarization observables for different pseudo-scalar meson photoproduction processes. For the first time, a complete or nearly complete measurement became possible and will facilitate model independent extraction of the reaction amplitude. An overview of the experimental program and its current status together with recent results on double polarization measurements in $\pi^{+}$photoproduction are presented.
\end{abstract}

\section{Introduction}

Among the most exciting and challenging topics in sub-nuclear physics today is the study of the structure of the nucleon and its different modes of excitation, the baryon resonances. Initially, most of the information on these excitations came primarily from partial wave analysis of data from $\pi N$ scattering. Recently, these data have been supplemented by a large amount of information from pion electroand photoproduction experiments. Yet, in spite of extensive studies spanning decades, many of the baryon resonances are still not well established and their parameters (i.e., mass, width, and couplings to various decay modes) are poorly known. Much of this is due to the complexity of the nucleon resonance spectrum, with many broad, overlapping resonances. While traditional theoretical approaches have highlighted a semi-empirical approach to understanding the process as proceeding through a multitude of $s$-channel resonances, $t$-channel processes, and non-resonant background, more recently attention has turned to approaches based on the underlying constituent quarks. An extensive review of the quark models of baryon masses and decays can be found in Ref. [1]. Most recently lattice QCD is making significant progress in calculations of baryon spectrum [2]. While these quark approaches are more fundamental and hold great promise, all of them predict many more resonances than have been observed, leading to the so-called "missing resonance" problem. One possible reason why they were not observed because they may have small coupling to the $\pi N$. At the same time they may have strong coupling to other final states $\eta N, \eta^{\prime} N, \mathrm{KY}, 2 \pi N$. The photo and electroproduction of various mesons is very attractive tool. While the significant amount of the meson photoproduction cross section measurements became available in recent years, these data alone cannot give an unambiguous answer about resonances. Since in pseudoscalar meson photoproduction there are three objects with spin involved: incident photon, target nucleon and recoil baryon. Therefore the process is descried by four spin dependent complex amplitudes. Total number of single and double polarization observables is sixteen. In order to be able to extract the amplitude of the process without any assumption one needs to measure at least eight carefully chosen single and double polarization observables.

A substantial part of the experimental efforts at the experimental Hall-B of Jefferson Laboratory is dedicated to this problem. In this report a general overview of the experimental capabilities in the

\footnotetext{
a e-mail: pasyuk@jlab.org
}

This is an Open Access article distributed under the terms of the Creative Commons Attribution License 2.0, which permits unrestricted use, distribution, and reproduction in any medium, provided the original work is properly cited. 
Experimental Hall-B will be presented followed by a few examples of recent double polarization experiments in pion photoproduction and finally overall status of the program.

\section{Experimental Hall-B}

Experimetal Hall-B at Jefferson Lab provides a unique set of instruments for these experiments. One instrument is the CLAS [3], a large acceptance spectrometer which allows detection of particles in a wide range of $\theta$ and $\phi$. The other instrument is a broad-range photon tagging facility [4] with the recent addition of the ability to produce linearly-polarized photon beams through coherent bremsstrahlung. The last but not least component essential for the double polarization experiments is a polarized target.

The Hall B photon tagger [4] covers a range in photon energies from 20 to $95 \%$ of the incident electron beam energy. Unpolarized, circularly polarized and linearly polarized tagged photon beams are presently available.

With a polarized electron beam incident on the bremsstrahlung radiator, a circularly polarized photon beam can be produced. The degree of circular polarization of the photon beam depends on the ratio $k=E_{\gamma} / E_{e}$. The magnitude of $P_{\odot}$ ranges from $60 \%$ to $99 \%$ of the incident electron beam polarization $P_{e}$ for photon energies $E_{\gamma}$ between $50 \%$ and $95 \%$ of the incident electron energy. CEBAF accelerator routinely delivers electron beam with polarization of $85 \%$ and higher.

A linearly polarized photon beam is produced by the coherent bremsstrahlung technique, using an oriented diamond crystal as a radiator. The degree of polarization is a function of the fractional photon beam energy and collimation and can reach $80 \%$ to $90 \%$. With linearly polarized photons, over $80 \%$ of the photon flux is confined to a $200-\mathrm{MeV}$ wide energy interval.

An essential piece of the hardware for this experiment is a polarized target capable of being polarized transversely and longitudinally with a minimal amount of material in the path of outgoing charged particles. These requirement are satisfied by a frozen spin target, FROST[5]. Since in the frozen spin mode the holding field is relatively low, it is possible to design a "transparent" holding magnet with a minimal amount of material for the charged particles to traverse on their way into CLAS. Butanol was chosen as target material. The target depolarization rate was about $1 \%$ a day. This allowed us to re-polarize the target once a week and maintain an average polarization of the order of $85 \%$.

With all these experimental tools in hands a "complete" experiment became possible.

\section{Double polarization measurements in $\pi^{+}$photoproduction}

Even though pion photoproduction is considered relatively well understood and "missing" resonances are expected to have weak coupling to $\pi N$ it is still important to get more constraints from polarization measurements for this reaction channel. Here we present the latest double polarization measurements of $\pi^{+}$photoproduction performed with circularly polarized beam and transversely polarized target.

For the transversely polarized target and circularly polarized photons the cross section can be written as:

$$
\frac{d \sigma}{d \Omega}=\left(\frac{d \sigma}{d \Omega}\right)_{0}\left(1+P_{t} T \sin (\phi)+P_{t} P_{\odot} F \cos (\phi)\right),
$$

where $\left(\frac{d \sigma}{d \Omega}\right)_{0}$ is unpolarized cross section, $P_{t}$ and $P_{\odot}$ are the degrees of target and circular beam polarization respectively, $T$ is single target asymmetry and $F$ is double spin asymmetry.

The preliminary results for $T$ and $F$ asymmetries are shown in Figs. 1 and 2. These results are based on a small fraction of statistics.

\section{Sumamry}

The present status of the meson phtotporduction program is shown in Table 1. There were several CLAS running periods with circularly and linearly polarized photon beams and unpolarized target. 

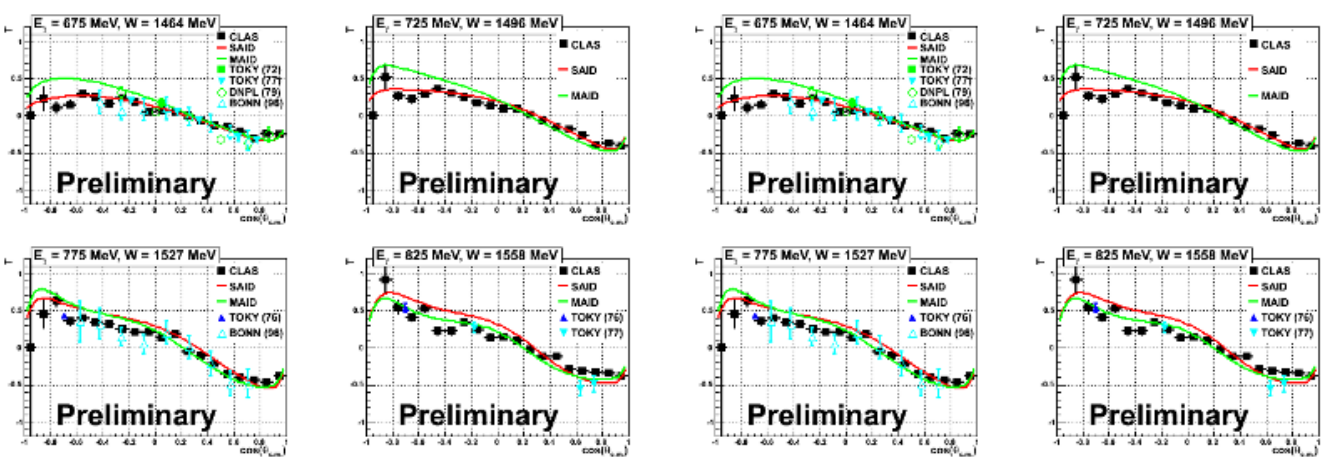

Fig. 1. Preliminary values of the T observable (black squares). Overlaid on these plots are the current SAID [10] (red), MAID2007 [7] (green) These data have not been included in the fits. Data analysis by M. Dugger, ASU.
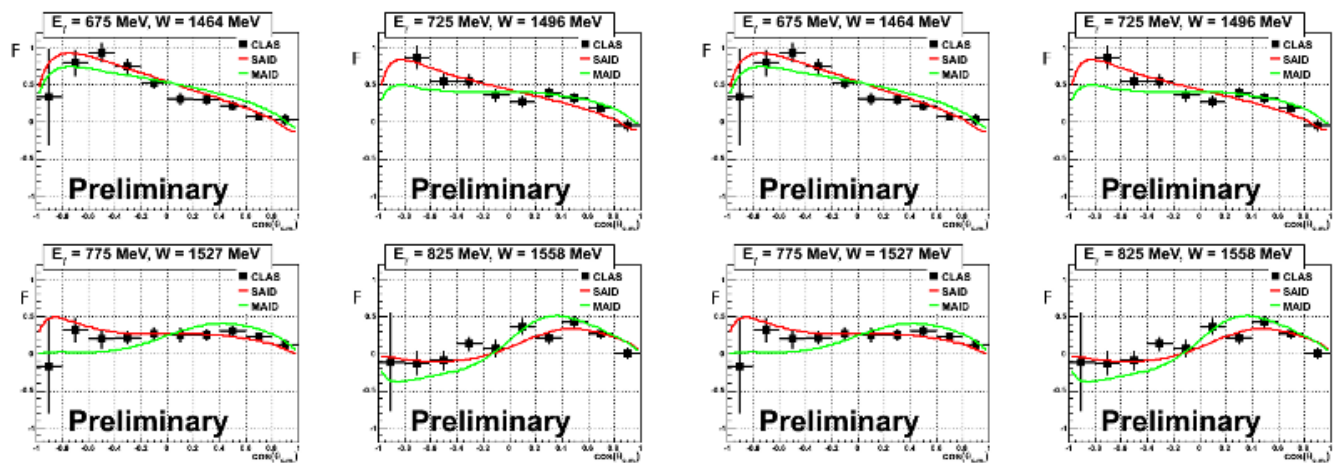

Fig. 2. Preliminary values of the F observable. Legend is the same as in Fig. 1. Data analysis by M. Dugger, ASU.

High quality data for the cross sections of $\pi^{0}[9], \pi^{+}[10], \eta[11,13], \eta^{\prime}[12,13]$ and kaon photoproduction were obtained. In addition to the cross sections for $K^{+} \Lambda[15,16,18]$ and $K^{+} \Sigma^{0}[15,16,19]$ final states the polarization of hyperons $P[15]$ and polarization transfer $C_{x} / C_{z}$ were measured[17]. The same final states have been studied with linearly polarized photons incident on unpolarized target. The analysis of the data from these experiments is in its final stage.

Once the frozen spin target became available we completed double polarization measurements with linearly and circularly polarized photons and longitudinally and transversely polarized proton target. The double polarization data are being analyzed. Due to self analyzing nature of the hyperon decays we have measured all 16 observables for $K Y$ final state. For non strange mesons we obtained nearly complete set of eight observables.

The double polarization measurement on the deuteron with polarized HD-Ice target were completed as well. As with a proton target we have complete set of observables for hyperon photoproduction. This is essential for understanding isospin structure of amplitude.

For the first time the complete measurements of meson photoproduction were performed at Jefferson Lab with CLAS. These measurements allow model independent reconstruction of the reaction amplitude which will be used for search and study of the nucleon excitation spectrum.

This work was supported in part by the Chilean Comisión Nacional de Investigación Científica y Tecnológica (CONICYT), the Istituto Nazionale di Fisica Nucleare, the French Centre National de la Recherche Scientifique, the French Commissariat à l'Energie Atomique, the U.S. Department of Energy, the National Science Foundation, the UK Science and Technology Facilities Council (STFC), the Scottish Universities Physics Alliance (SUPA), and the National Research Foundation of Korea.

Authored by Jefferson Science Associates, LLC under U.S. DOE Contract No. DE-AC05-06OR231 77. The U.S. Government retains a non-exclusive, paid-up, irrevocable, world-wide license to publish or reproduce this manuscript for U.S. Government purposes. 
Table 1. Observables in single pseudo-scalar meson photoproduction. " $\checkmark$ "indicates data already accumulated and being analyzed.

\begin{tabular}{|c|c|c|c|c|c|c|c|c|c|c|c|c|c|c|c|c|}
\hline & $\sigma$ & $\Sigma$ & $T$ & $P$ & $E$ & $F$ & $G$ & $H$ & $T_{x}$ & $T_{y}$ & $L_{x}$ & $L_{z}$ & $O_{x}$ & $O_{z}$ & $C_{x}$ & $C_{z}$ \\
\hline \multicolumn{17}{|c|}{ Proton target } \\
\hline$p \pi^{0}$ & [9] & $\checkmark$ & $\checkmark$ & $\checkmark$ & $\checkmark$ & $\checkmark$ & $\checkmark$ & $\checkmark$ & & & & & & & & \\
\hline$n \pi^{+}$ & [10] & $\checkmark$ & $\checkmark$ & $\checkmark$ & $\checkmark$ & $\checkmark$ & $\checkmark$ & $\checkmark$ & & & & & & & & \\
\hline$p \eta$ & {$[11,13]$} & $\checkmark$ & $\checkmark$ & $\checkmark$ & $\checkmark$ & $\checkmark$ & $\checkmark$ & $\checkmark$ & & & & & & & & \\
\hline$p \eta \prime$ & {$[12,13]$} & $\checkmark$ & $\checkmark$ & $\checkmark$ & $\checkmark$ & $\checkmark$ & $\checkmark$ & $\checkmark$ & & & & & & & & \\
\hline$p \omega$ & [14] & $\checkmark$ & $\checkmark$ & $\checkmark$ & $\checkmark$ & $\checkmark$ & $\checkmark$ & $\checkmark$ & & & & & & & & \\
\hline$K^{+} \Lambda$ & {$[15,16,18]$} & $\checkmark$ & $\checkmark$ & {$[15,18]$} & $\checkmark$ & $\checkmark$ & $\checkmark$ & $\checkmark$ & $\checkmark$ & $\checkmark$ & $\checkmark$ & $\checkmark$ & $\checkmark$ & $\checkmark$ & [17] & [17] \\
\hline$K^{+} \Sigma^{0}$ & {$[15,16,19]$} & $\checkmark$ & $\checkmark$ & {$[15,19]$} & $\checkmark$ & $\checkmark$ & $\checkmark$ & $\checkmark$ & $\checkmark$ & $\checkmark$ & $\checkmark$ & $\checkmark$ & $\checkmark$ & $\checkmark$ & [17] & [17] \\
\hline \multicolumn{17}{|c|}{ Neutron (deuteron) target } \\
\hline$p \pi^{-}$ & [20] & $\checkmark$ & & & $\checkmark$ & & $\checkmark$ & & & & & & & & & \\
\hline$K^{-} \Sigma^{+}$ & $\checkmark$ & $\checkmark$ & & & $\checkmark$ & & $\checkmark$ & & & & & & & & & \\
\hline$K^{0} \Lambda$ & $\checkmark$ & $\checkmark$ & $\checkmark$ & $\checkmark$ & $\checkmark$ & $\checkmark$ & $\checkmark$ & $\checkmark$ & $\checkmark$ & $\checkmark$ & $\checkmark$ & $\checkmark$ & $\checkmark$ & $\checkmark$ & $\checkmark$ & $\checkmark$ \\
\hline$K^{0} \Sigma^{0}$ & $\checkmark$ & $\checkmark$ & $\checkmark$ & $\checkmark$ & $\checkmark$ & $\checkmark$ & $\checkmark$ & $\checkmark$ & $\checkmark$ & $\checkmark$ & $\checkmark$ & $\checkmark$ & $\checkmark$ & $\checkmark$ & $\checkmark$ & $\checkmark$ \\
\hline
\end{tabular}

\section{References}

1. S. Capstick and W. Roberts, Prog. Part. Nucl. Phys. (Suppl. 2) 45, (2000) S241.

2. R. G. Edwards, J. J. Dudek, D. G. Richards, and S. J. Wallace, arXiv:1104.5152.

3. B.A. Mecking et al. (CLAS Collaboration), Nucl. Inst. Meth. A 503, (2003) 513.

4. D.I. Sober et al., Nucl. Inst. Meth. A 440, (2000) 263.

5. C.D. Keith et al., Nucl. Inst. Meth. A 684, (2012) 27.

6. J. McAndrew, D. Watts, E. Pasyuk in: Proceedings of NSTAR 2011 - The 8th International Workshop on the Physics of Excited Nucleons, May 17-20, 2011, Thomas Jefferson National Accelerator Facility, Newport News, Virginia USA

7. D. Drechsel, O. Hanstein, S. S. Kamalov, L, Tiator, Nucl. Phys. A465, (1999) 145.

8. E. Klempt, A. V. Anisovich, V. A. Nikonov, A. V. Sarantsev, U. Thoma, Eur. Phys. J. A 24 (2005) 111.

9. M. Dugger et al. (The CLAS Collaboration), Phys. Rev. C 76 (2007) 025211.

10. M. Dugger et al. (The CLAS Collaboration), Phys. Rev. C 79 (2009) 065206.

11. M. Dugger et al. (The CLAS Collaboration), Phys. Rev. Lett. 89 (2002) 222002.

12. M. Dugger et al. (The CLAS Collaboration), Phys. Rev. Lett. 96 (2006) 062001, Erratum-ibid. 96: 169905.

13. M. Williams et al. (The CLAS Collaboration), Phys. Rev. C 80, (2009) 045213.

14. M. Williams et al. (The CLAS Collaboration), Phys. Rev. C 80, (2009) 065209.

15. J. W. C. McNabb et al. (The CLAS Collaboration), Phys. Rev. C 69, (2004) 042201.

16. R. Bradford et al. (The CLAS Collaboration), Phys. Rev. C 73 (2006) 035202.

17. R. Bradford et al. (The CLAS Collaboration), Phys. Rev. C 75, (2007) 035205.

18. M. E. McCracken et al. (The CLAS Collaboration), Phys. Rev. C 81, (2009) 025201.

19. B. Dey et al. (The CLAS Collaboration), Phys. Rev. C 82, (2010) 025202.

20. W. Chen (The CLAS Collaboration), Phys. Rev. Lett. 103, (2009) 012301. 